\title{
Improving mechanical properties of high volume fraction aligned multi-walled carbon nanotube/epoxy composites by stretching and pressing
}

\author{
Tran Huu Nam ${ }^{a^{*}}$, Ken Goto ${ }^{\mathrm{b}}$, Yudai Yamaguchi ${ }^{\mathrm{c}}$, E.V.A. Premalal ${ }^{\mathrm{c}}$, Yoshinobu \\ Shimamura $^{\mathrm{c}}$, Yoku Inoue ${ }^{\mathrm{c}}$, Shuichi Arikawa ${ }^{\mathrm{d}}$, Satoru Yoneyama ${ }^{\mathrm{d}}$, Shinji Ogihara ${ }^{\mathrm{e}}$ \\ ${ }^{a}$ Faculty of Fundamental Sciences, Petrovietnam University, Long Toan, Ba Ria, Ba Ria-Vung Tau 790000, Viet Nam \\ ${ }^{\mathrm{b}}$ Department of Space Flight Systems, Institute of Space and Astronautical Science, Japan Aerospace Exploration \\ Agency, 3-1-1 Yoshinodai, Chuo, Sagamihara, Kanagawa 252-5210, Japan

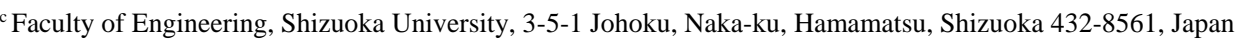 \\ ${ }^{d}$ Department of Mechanical Engineering, Aoyama Gakuin University, 5-10-1 Fuchinobe, Chuo, Sagamihara, Kanagawa 252-5258, \\ Japan \\ ${ }^{\mathrm{e}}$ Department of Mechanical Engineering, Tokyo University of Science, 2641 Yamazaki, Noda, Chiba 278-8510, Japan
}

Corresponding author: Fax: +81 (0)42-759-8532

E-mail addresses: tran.huunam@ac.jaxa.jp; namth@pvu.edu.vn (Tran Huu Nam)

\section{ABSTRACT}

Aligned multi-walled carbon nanotube (CNT) sheets produced from aligned CNT arrays were used to develop high volume fraction CNT/epoxy composites. Stretching and pressing techniques were applied during CNT sheet processing to straighten the wavy CNTs and to enhance the dense packing of CNTs in the sheets. Raman spectra measurements showed better CNT alignment in the CNT sheets and the composites after stretching and pressing. Aligned CNT/epoxy composites with CNT volume fraction up to $63.4 \%$ were developed using hot-melt prepreg processing with a vacuumassisted system. Stretching and pressing of the CNT sheets enhanced the mechanical properties of high volume fraction CNT/epoxy composites considerably. Stretching and pressing increased tensile strength of the composites by $32 \%$ and elastic modulus of the 
composites by $27 \%$. Applying stretching and pressing is effective for production of superior CNT sheets with high alignment and dense packing of CNTs, thereby supporting the development of high-performance CNT composites.

Keywords: A. Polymer-matrix composites (PMCs); A. Nano-structures; B. Mechanical properties; D. Mechanical testing.

\section{Introduction}

Carbon nanotubes (CNTs) have highly desirable mechanical, electrical, and thermal properties [1-4]. Their excellent mechanical properties along with their low density make CNTs attractive as a potential reinforcement material for next-generation advanced composites. The advanced composites used for aerospace structures comprise a high volume fraction of aligned stiff fibers embedded in high-performance polymers [5]. Vertically aligned CNT arrays have been developed for the production of high volume fraction aligned CNT-reinforced polymer composites [6-9]. Furthermore, Wardle et al. [10] fabricated high volume fraction aligned CNT/epoxy composites using mechanical densification of vertically aligned CNT arrays, followed by capillarityinduced wetting with unmodified epoxies. However, the composite length was restricted drastically because of limited height of the CNT arrays. Therefore, long-aligned CNT sheets have been created recently from vertically aligned CNT arrays using solid-state drawing and winding techniques [11-13]. Highly oriented aligned CNT sheets have been particularly promising for the development of high volume fraction CNT composites with high performance.

High volume fraction CNT composites based on aligned CNT sheets have attracted great interest because they are envisioned as a revolutionary advanced composite 
material for a host of demanding applications [14-17]. The high volume fraction allows the properties of CNTs to dominate the composite properties [10]. However, several reports have described that the waviness, entanglement, and poor packing of CNTs in the sheets degraded the mechanical properties of their composites [18-22]. Therefore, mechanical stretching has been applied to the CNT sheets to straighten the wavy CNTs and to enhance dense packing of CNTs, thereby improving the strength and stiffness of CNT-reinforced composites [23-25]. Nevertheless, the handling of the CNT sheets without resin for mechanical stretching is generally difficult because of static electricity [15]. Consequently, Nam et al. [26] proposed a simple press-drawing process by which pressing was applied directly where a CNT web enters the winding roll to create superior aligned CNT sheets with high strength and stiffness. These CNT sheets are effective for the production of high-performance CNT composites.

Recently, a stretch-winding technique has been applied to fabricate high volume fraction CNT-reinforced polymer composites with high strength and stiffness [16]. For this study, aligned CNT sheets were produced from vertically aligned CNT arrays using a novel combination of stretch-drawing and press-winding techniques. These techniques can reduce the waviness and entanglement of CNTs considerably, and can increase dense packing of CNTs in the sheets, thereby improving the properties of aligned CNT composites. High volume fraction CNT composites based on epoxy resin and aligned CNT sheets were developed using hot-melt prepreg processing with a vacuum-assisted system (VAS). The mechanical properties of the high volume fraction CNT/epoxy composites were studied. The CNT volume fraction was estimated through thermogravimetric analysis (TGA) data. Field emission scanning electron microscopy (FE-SEM) (SU8030; Hitachi Ltd., Tokyo, Japan) was used to investigate the microstructural morphologies of the CNT sheets and their composites. 


\section{Experimental procedures}

\subsection{Materials}

A B-stage epoxy resin sheet covered with release paper and plastic film was obtained from Sanyu Rec Co. Ltd. (Osaka, Japan) with the recommended cure condition of $130^{\circ} \mathrm{C}$ for $2 \mathrm{~h}$. The areal weight of the B-stage epoxy resin sheet with density of 1.2 $\mathrm{g} / \mathrm{cm}^{3}$ was controlled to approximately $12 \mathrm{~g} / \mathrm{m}^{2}$. Vertically aligned multi-walled CNT arrays with about $0.8 \mathrm{~mm}$ height were grown on a bare quartz substrate using chloridemediated chemical vapor deposition [7]. Fig. 1a portrays a vertically aligned CNT array used for this study. An FE-SEM micrograph showing horizontally aligned CNTs drawn from the CNT array was inserted in Fig. 1a. A scanning transmission electron microscopy (STEM) image showing the high quality of CNTs is presented in Fig. 1b. As-grown CNTs examined in this study have mean diameter of $22 \mathrm{~nm}$ (see Fig. $1 \mathrm{~b}$ inset).

\subsection{Processing of aligned CNT sheets}

The main purpose of our strategy for fabricating high-strength and high-modulus CNT composites is to create superior aligned CNT sheets before embedding them into a polymer matrix. Pristine aligned CNT sheets have been produced from vertically aligned CNT arrays using drawing and winding processes $[12,15]$. Although most of the CNTs are aligned unidirectionally, many wavy and entangled CNTs were observed in the pristine CNT sheets. Therefore, mechanical stretching has been applied to the aligned CNT sheets to straighten the wavy CNTs in the sheets [25]. In addition, a stretching system to stretch CNT webs during the CNT sheet processing has been reported recently by Wang et al. [16]. The CNT webs travelled horizontally and passed through a stretching system including a pair of stationary rods. For our study, stretched 
CNT sheets were produced from aligned CNT arrays through drawing and stretchwinding processes. Furthermore, pressed CNT sheets were created from aligned CNT arrays using drawing and press-winding techniques, as presented by Nam et al. [26]. Moreover, a new combination of both stretching and pressing was proposed to develop stretch-pressed CNT sheets for additional improvement of the composite properties. Fig. 2 depicts a schematic showing the processing of an aligned CNT sheet using drawing, stretching, winding, and pressing techniques. Pristine, stretched, pressed, and stretchpressed 300-ply aligned CNT sheets were used for composite fabrication.

\subsection{Production of aligned CNT/epoxy composites}

Composites made of an epoxy resin film and aligned CNT sheets were developed using hot-melt prepreg processing with the VAS. This method maintained the alignment of CNTs during epoxy resin impregnation [25,26]. First, an aligned CNT/epoxy prepreg was prepared by stacking 300-ply CNT sheets with $20 \mathrm{~mm}$ width and $40 \mathrm{~mm}$ length on an epoxy resin film. Then, the prepreg was set between two release films (WL5200; Airtech International Inc., CA, USA). Next, the prepreg was pressed under $0.5 \mathrm{MPa}$ pressure for 5 min at $100^{\circ} \mathrm{C}$ using a test press (Model MP-WNL; Toyo Seiki SeisakuSho Ltd., Tokyo, Japan). Subsequently, the prepregs were peeled from the release films and release paper. Finally, the prepregs were cured at $130^{\circ} \mathrm{C}$ for $2 \mathrm{~h}$ under $2 \mathrm{MPa}$ in the VAS to produce the composites. The pristine, stretched, pressed, and stretch-pressed CNT/epoxy composites were fabricated for comparative assessments.

\subsection{Thermogravimetric analysis}

The thermal degradation behaviors of epoxy resin, CNTs, and their composites were analyzed up to $800^{\circ} \mathrm{C}$ in $\operatorname{argon}$ gas at a flow rate of $300 \mathrm{ml} / \mathrm{min}$ using a thermogravimetric analyzer (DTG-60A; Shimadzu Corp., Kyoto, Japan). About 5 mg of 
each specimen was loaded for each measurement at a heating rate of $10^{\circ} \mathrm{C} / \mathrm{min}$. The respective mass losses of epoxy resin, CNTs, and the composites were recorded.

\subsection{Characterizations and testing}

Polarized Raman spectra were measured to ascertain the degree of CNT alignment in the sheets and their composites using Raman spectroscope with laser excitation of 532 nm (XploRA-ONE; Horiba Ltd., Kyoto, Japan). Tensile tests were conducted for the aligned CNT/epoxy composites in a laboratory environment at room temperature (RT) of $23 \pm 3^{\circ} \mathrm{C}$ and $50 \pm 5 \%$ relative humidity. Tensile specimens with $10 \mathrm{~mm}$ gauge length and $3 \mathrm{~mm}$ width were tested on a testing machine (EZ-L; Shimadzu Corp., Kyoto, Japan) with a crosshead speed of $0.1 \mathrm{~mm} / \mathrm{min}$. Widths of specimens were measured using an optical microscope (SZX12; Olympus Corp., Tokyo, Japan), whereas their thickness was measured using a micrometer (102-119; Mitutoyo Corp., Kanagawa, Japan). The longitudinal strain of tensile specimens was measured using a noncontacting video extensometer (TRViewX; Shimadzu Corp., Kyoto, Japan) with two targets. Mean tensile properties were obtained from at least five specimens.

\section{Results and discussion}

\subsection{Evaluation of CNT alignment after stretching and/or pressing}

FE-SEM micrographs showing microstructural morphologies of pristine, stretched, pressed, and stretch-pressed CNT sheets are presented in Fig. 3. The pristine CNT sheets showed many wavy and entangled CNTs (Fig. 3a). The waviness and entanglement of numerous CNTs in the sheets were reduced slightly after the CNT webs traveling throughout the stretching system (Fig. 3b). The CNT waviness reduction is explainable by the increased tension of the CNT web when it passes around a pair of stationary rods [16]. However, the packing of CNTs in the pristine sheets and stretched 
sheets is poor. Therefore, pressing was applied to the pristine sheets and stretched sheets to reduce the waviness of CNTs further and especially to enhance the dense packing of CNTs in the sheets. After pressing, the waviness of CNTs in the sheets diminished considerably (Figs. 3c-3d). The reduced waviness of CNTs attributable to pressing is explainable through the mechanism of press load-induced tension in winding [26,27]. Particularly, the pressing drastically enhances the dense packing of CNTs in the sheets (see Figs. 3c-3d). Moreover, the stretch-pressed CNT sheets (Fig. 3d) showed more straight CNTs and greater CNT alignment than the pressed CNT sheets (Fig. 3c).

The CNT alignment and straightening of wavy CNTs after stretching and/or pressing were examined using polarized Raman spectroscopy [28,29]. Polarized Raman spectra were measured using incident light (laser light with a wavelength of $532 \mathrm{~nm}$ ) normal to the CNT sheet samples. The incident light was polarized parallel and perpendicular to the CNT alignment (see Fig. 4 inset). Typical Raman spectra with Raman shift between $1000-2000 \mathrm{~cm}^{-1}$ are presented in Fig. 4. Polarized Raman spectra for all samples show two main peaks located at approximately $1350 \mathrm{~cm}^{-1}$ and $1580 \mathrm{~cm}^{-1}$, which are attributed respectively to the disorder-induced $\mathrm{D}$ band and the graphite-structure derived $\mathrm{G}$ band. The Raman shift of the CNT sheets does not change significantly after stretching and/or pressing. Furthermore, the intensity ratio between the $\mathrm{G}$ band and the $\mathrm{D}$ band $\left(I_{G} / I_{D}\right)$ of the CNT sheets is higher than 3.0. This high value shows the high crystal quality of CNTs and the low amount of amorphous carbon, as presented by Inoue et al. [12].

In other respects, the ratio of G-band intensity in the parallel configuration to the perpendicular configuration $\left(R=I_{G \|} / I_{G \perp}\right)$ was used to characterize the degree of CNT alignment $[12,28,29]$. The higher CNT alignment produces the higher G-band intensity ratio because Raman scattering is more intense when the polarization of the incident 
light is parallel to the axis of a CNT [30]. The G-band intensity ratio $R$ of the pristine CNT sheets was 1.73, as portrayed in Fig. 4. After stretching and/or pressing, the $R$ value of CNT sheets was markedly enhanced. The respective G-band intensity ratios of the stretched and pressed CNT sheets were 2.57 and 4.82. It is particularly interesting that the $R$ value of the stretch-pressed CNT sheets increased drastically to 6.30. The marked enhancement in the $R$ is attributed to the better alignment of CNTs in the sheets after stretching and/or pressing. Results show that the G-band intensity ratio of the stretch-pressed samples is much greater than that of the pristine ones. Therefore, the combination of both stretching and pressing improved the CNT alignment in the sheets considerably.

\subsection{CNT volume fraction in the composites}

The CNT volume fraction was determined through the TGA data. The respective mass losses of the CNTs, epoxy resin and the composites were measured at $150-750^{\circ} \mathrm{C}$. The CNT mass fraction $\left(m_{f}\right)$ of the composite was calculated from the mass loss of the CNTs $\left(\Delta m_{f}\right)$, epoxy resin $\left(\Delta m_{m}\right)$ and the composite $\left(\Delta m_{c}\right)$ as follows.

$$
m_{f}=\frac{\left(\Delta m_{m}-\Delta m_{c}\right)}{\left(\Delta m_{m}-\Delta m_{f}\right)}
$$

The CNT volume fraction $\left(V_{f}\right)$ was then estimated from the mass fraction of the CNTs, epoxy resin density $\left(\rho_{m}\right)$, and the density of the composite $\left(\rho_{c}\right)$ as follows.

$$
V_{f}=1-\frac{\left(1-m_{f}\right) \rho_{c}}{\rho_{m}}
$$

The mass losses, CNT mass fractions, and CNT volume fractions of the composites are presented in Table 1. The stretching and/or pressing of the CNT sheets induced a slight enhancement in the CNT mass fraction and the CNT volume fraction of the composites. 
The increase in the CNT volume fraction of the composites was explained by the decrease of the composite thickness $[25,26]$. The composite thickness reduction is attributable to straightening of the wavy CNTs and dense packing of CNTs in the sheets caused by the stretching and/or pressing (see Fig. 3). Particularly, the combination of both stretching and pressing of the CNT sheets drastically engendered the straightening of wavy CNTs and denser packing of CNTs in the composites.

\subsection{Mechanical properties of high volume fraction CNT/epoxy composites}

The mechanical properties of epoxy resin and high volume fraction aligned CNT/epoxy composites were measured using tensile testing. The mean tensile strength, elastic modulus and fracture strain of epoxy resin respectively were 64.4 MPa, 2.6 GPa, and 4.8\%. The composites showed a linear stress-strain relation until the specimen fractures with no bending of the curves at high loads. Mechanical properties of pristine, stretched, pressed, and stretch-pressed CNT/epoxy composites are presented in Table 2. FE-SEM micrographs showing in-plane distribution of CNTs in the pristine, stretched, pressed, and stretch-pressed aligned CNT/epoxy composites are depicted in Fig. 5. The wavy and poor-packed CNTs are apparent in the pristine composites (Fig. 5a). During stretching and/or pressing, the wavy CNTs were straightened and the dense packing of CNTs was enhanced (see Figs. 5b-5d). High-magnification FE-SEM micrographs showing fracture surfaces of the composites are presented in Fig. 6. As observed in Fig. 6, CNT bundles created by the stretching and/or pressing are visible on the fracture surfaces of the composites. The existence of the CNT bundles is evidenced by the surface morphologies of the CNT sheets (Figs. 3b-3d).

Compared with epoxy resin, the pristine aligned CNT/epoxy composite showed increased tensile strength by $821 \%$, enhanced elastic modulus by $4042 \%$, and decreased fracture strain by $88 \%$. The improvement in tensile strength and elastic modulus of the 
composites is explainable by the fact that aligned CNTs in the composites carry the load along the length of CNTs and provide strength and stiffness in the loading direction [25,26]. As Fig. 3 shows, most CNTs in the sheets are well aligned in the drawing direction. In addition, the alignment of CNTs in the composites was maintained during resin impregnation using the hot-melt prepreg processing (see Fig. 5). The reduction in the fracture strain of the composites is mainly attributable to the addition of high CNT contents, leading to the decrease in the amount of epoxy matrix available for the elongation, as presented in our earlier reports $[25,26]$.

Applying stretching and/or pressing of the CNT sheets improved the mechanical properties of the high volume fraction CNT/epoxy composites (see Table 2). Compared with the pristine CNT/epoxy composites, the stretched composites showed tensile strength enhancement of $14 \%$ and elastic modulus increase of $12 \%$. The enhancement in tensile strength and elastic modulus is attributed to the straightening of wavy CNTs (see Fig. 3b) and the slight increase of CNT volume fraction (Table 1). The wavy CNTs were observed clearly in the pristine samples (Fig. 3a and Fig. 5a). When the CNT webs passed through a stretching system during the CNT sheet processing (see Fig. 2), the wavy CNTs were partly straightened along the stretch direction. The straightening of wavy CNTs is explainable by the fact that the CNT web tension was enhanced by the stretching system [16]. The straightening of wavy CNTs in Fig. 3b caused by the stretching system enhanced the mechanical properties of the stretched CNT/epoxy composites.

In addition, the pressed CNT/epoxy composites showed superior mechanical properties to those of the pristine and stretched ones (see Table 2). This enhancement is attributable to greater straightening of wavy CNTs and especially to the increase of CNT dense packing (Fig. 3 and Fig. 5). As described above, the straightening of wavy 
CNTs caused by pressing is attributable to the mechanism of press load-induced tension in winding. In press-winding method, radial pressure is applied to the CNT sheet at the point where the CNT web enters the winding spool. This pressure increases the tension of the CNT webs in the CNT sheet, thereby leading to the straightening of wavy CNTs [26]. Furthermore, when the CNT webs are pressed during the CNT sheet processing, the CNTs in the pressed sheets are packed densely with fewer interstices between the CNTs (see Fig. 3). The dense packing of CNTs in the pressed composites became more compact than that in the stretched composites. Therefore, pressing without stretching the CNT sheets is more effective in improving the mechanical properties of the composites than stretching without pressing.

Moreover, the combination of both stretching and pressing of the CNT sheets improved the mechanical properties of the composites considerably. The stretch-pressed aligned CNT/epoxy composites respectively exhibited increased tensile strength by $32 \%$, $16 \%$, and $8 \%$, and increased elastic modulus by $27 \%, 13 \%$, and $6 \%$ compared with the pristine, stretched, and pressed ones. Application of both stretching and pressing evidently enhanced the straightening of wavy CNTs and simultaneously increased dense packing of CNTs (see Fig. 3d and Fig. 5d). The increase of alignment and dense packing of CNTs enhanced the strength and stiffness of the aligned CNT/epoxy composites [26]. In general, the pristine composites contained many wavy CNTs along the axial loading direction and indicated poor-packed CNTs. Therefore, just a portion of CNT fraction in the composites carries loads effectively in the tensile testing. Applying stretching and/or pressing produced the straightening of wavy CNTs and created many CNT bundles (see Fig. 3 and Fig. 6). The straightened CNTs have a larger fraction of 
their length aligned with the loading direction, thereby improving the mechanical strength and stiffness of the composites [25].

\subsection{Evaluating the increase in CNT alignment and volume fraction}

Improving the mechanical properties of aligned CNT/epoxy composites originated from the straightening of wavy CNTs and from the dense packing of CNTs, which are ascribed to the stretching and/or pressing. The dense packing of CNTs in the sheets caused the increased CNT volume fraction in the composites [25,26]. In addition, the straightening of wavy CNTs by stretching and/or pressing engenders the increase of the G-band intensity ratio $R$. For the composite specimens, the intensity ratio between the G-bands and D-bands and the G-band intensity ratio for the two polarizations are presented in Table 3. Results show that the intensity ratio $I_{G} / I_{D}$ of the composites varies slightly with change of the CNT sheet processing. However, the G-band intensity ratio of the composite samples is increased markedly when applying stretching and/or pressing. The G-band intensity enhancement is attributed to the better alignment of CNTs caused by stretching and/or pressing of the CNT sheets, as described above. The G-band intensity ratio of the stretch-pressed composite exhibited the highest value among all the composites. Consequently, in this study the combination of stretching and pressing indicated the best alignment of CNTs in the composites, as shown in Fig. 5.

As presented above, the G-band intensity ratio is used to investigate the degree of CNT alignment. The straightening of wavy CNTs by stretching and/or pressing produces a higher degree of CNT alignment, thereby leading to a higher G-band intensity ratio. To evaluate the increased straightening of wavy CNTs caused by the stretching and/or pressing, the relations of $\sigma / V_{f}$ (tensile strength/volume fraction) and of $E / V_{f}$ (elastic modulus/volume fraction) versus the G-band intensity ratio were 
analyzed, yielding the results presented in Fig. 7. Results show that the values of $\sigma / V_{f}$ and $E / V_{f}$ increased concomitantly with enhancement of the G-band intensity ratio. The enhancement in the $\sigma / V_{f}$ and $E / V_{f}$ with increasing the G-band intensity ratio is attributable to the increased straightening of wavy CNTs caused by the stretching and/or pressing. The increased straightening of wavy CNTs which arises from the stretching and/or pressing can be observed in Fig. 3 and Fig. 5.

Moreover, to quantify the straightening of wavy CNTs caused by the stretching and pressing, the CNT orientations in FE-SEM images taken from polished surfaces of the pristine and stretch-pressed composite samples were analyzed, as presented by Nam et al. [26]. The FE-SEM images were divided into five parts in the vertical direction, as depicted in Fig. 8 inset. The local orientation angle of CNTs was evaluated from each part with the assumption of straight CNTs. The histograms of local orientation angles of CNTs for six FE-SEM images of each pristine and stretch-pressed composite group were obtained. The individual histograms from the images in each group were summed up to calculate the global orientation of CNTs for both the pristine and stretch-pressed composites. Histograms to visualize the percentage frequency distribution of CNTs with global orientation angles between $-90 \mathrm{deg}$ and $+90 \mathrm{deg}$ with respect to the axial direction of CNTs in the pristine and stretch-pressed composites are presented in Fig. 8.

As observed in Fig. 8, the frequency of CNTs of the pristine composites distributes largely between -90 deg and +90 deg, whereas most CNTs in the stretch-pressed composites are aligned around the 0 deg direction (tensile direction). The standard deviation of the CNT orientation is about $40 \mathrm{deg}$ for the pristine composites, but it is reduced to $29 \mathrm{deg}$ for the stretch-pressed composites. Compared with the pristine composites, the stretch-pressed composites showed a slight decrease in the percentage 
frequency of CNTs from $-90 \mathrm{deg}$ to $-45 \mathrm{deg}$ and between $45 \mathrm{deg}$ and $90 \mathrm{deg}$. The slight reduction indicates that the entangled CNTs in the composites decrease only slightly after applying the stretching and pressing. Furthermore, the percentage frequency of CNTs with orientation angles from $-45 \mathrm{deg}$ to $-15 \mathrm{deg}$ and between $15 \mathrm{deg}$ and $45 \mathrm{deg}$ in the pristine composites was higher than that in the stretch-pressed ones. Therefore, the frequency distribution of CNTs with orientation angles between $-15 \mathrm{deg}$ and $15 \mathrm{deg}$ increases from $28 \%$ for the pristine composites to $63 \%$ for stretch-pressed composites. This increase is attributed to the straightening of wavy CNTs in the stretch-pressed composites resulting from the stretching and pressing.

The stretching and pressing of the CNT sheets markedly increased the mechanical properties of the composites. As described above, the enhanced mechanical properties of the composites are attributable to the increase of the CNT volume fraction caused by dense packing of CNTs and to the straightening of wavy CNTs. To evaluate the influence of these two factors, the respective percent increases of tensile strength, elastic modulus, and the CNT volume fraction of the stretched, pressed, and stretch-pressed composites compared with those of the pristine composites were analyzed, yielding the results presented in Fig. 9. Results show that the percentage increases in the tensile strength, elastic modulus, and the CNT volume fraction of the pressed composites are higher than those of the stretched ones. Therefore, the effectiveness of the pressing without stretching in improving tensile strength and elastic modulus of the composites is greater than that of the stretching without pressing. Moreover, the combination of both stretching and pressing showed the highest percentage increase in the tensile strength and elastic modulus of the composites. Consequently, the application of both stretching and pressing in the CNT sheet processing is most effective to create superior 
CNT sheets with high CNT alignment and dense packing of CNTs for the development of high-performance CNT-reinforced composites.

The percentage increases of the CNT volume fraction are evidently lower than those of tensile strength and elastic modulus of the composites (see Fig. 9). The percentage enhancement of tensile strength and elastic modulus as a result of increasing the CNT volume fraction was recognized as lower than that coming from the straightening of wavy CNTs $[25,26]$. Therefore, the volume fraction increase of the composites with high CNT loading was probably less efficient than the straightening of wavy CNTs resulting from the stretching and pressing. Moreover, the percentage increase of the elastic modulus (19.5\%) caused by pressing for high CNT volume fraction composites in this study was lower than that (77.6\%) for low volume fraction composites reported by Nam et al. [26]. This phenomenon demonstrates a reduced trend of the percentage increase in the elastic modulus of the composites as the CNT volume fraction increases. This decreased trend is attributable to the increase of CNT alignment in the thickness direction of the pristine composites with high CNT loading because the CNT orientation was distributed both over a plane direction and thickness direction of the composites [31]. In general, the efficiency of the stretching and/or pressing in improving the mechanical properties of the composites decreased with the increase of the CNT volume fraction.

\section{Conclusions}

Stretching and pressing techniques have produced superior CNT sheets with high alignment and dense packing of CNTs. Raman spectra measurements showed better CNT alignment in the CNT sheets and their composites after stretching and/or pressing. The aligned CNT/epoxy composites with high CNT volume fraction of $63.4 \%$ were 
developed successfully using hot-melt prepreg processing with the VAS. The stretching and/or pressing of the CNT sheets improved the mechanical properties of the composites considerably. Pressing without stretching is more effective than stretching without pressing. The highest strength and stiffness of the composites were achieved in the case of combining both stretching and pressing of the CNT sheets. The stretchpressed composites exhibit increased tensile strength by $32 \%$ and enhanced elastic modulus by $27 \%$ compared with pristine composites. Maximum tensile strength and elastic modulus of the aligned CNT/epoxy composites respectively reached as high as $851 \mathrm{MPa}$ and $147 \mathrm{GPa}$. In conclusion, the new combination of both stretching and pressing is most effective to produce superior CNT sheets for the development of high volume fraction CNT composites with high strength and stiffness.

\section{Acknowledgements}

We appreciate financial support from the Japan Science and Technology Agency (JST) through the Advanced Low Carbon Technology Research and Development Program (ALCA) and the Institute of Space and Astronautical Science (ISAS) through the ISAS strategic development fund for space engineering. This research is also funded by Petrovietnam University under grant code GV1514.

\section{References}

[1] Ruoff RS, Lorents DC. Mechanical and thermal properties of carbon nanotubes. Carbon 1995;33(7):925-930.

[2] Treacy MMJ, Ebbesen TW, Gibson JM. Exceptionally high Young's modulus observed for individual carbon nanotubes. Nature 1996;381:678-680.

[3] Salvetat JP, Kulik AJ, Bonard JM, Forro L, Benoit W, Zuppiroli L. Mechanical properties of carbon nanotubes. Appl Phys A 1999;69(3):255-260. 
[4] Ebbesen TW, Lezec HJ, Hiura H, Bennett JW, Ghaemi HF, Thio T. Electrical conductivity of individual carbon nanotubes. Nature 1996;382:54-56.

[5] Cebeci H, de Villoria RG, Hart AJ, Wardle BL. Multifunctional properties of high volume fraction aligned carbon nanotube polymer composites with controlled morphology. Compos Sci Technol 2009;69(15-16):2649-2656.

[6] Xiong GY, Wang DZ, Ren ZF. Aligned millimeter-long carbon nanotube arrays grown on single crystal magnesia. Carbon 2006;44(5):969-973.

[7] Inoue Y, Kakihata K, Hirono Y, Horie T, Ishida A, Mimura H. One-step grown aligned bulk carbon nanotubes by chloride mediated chemical vapor deposition. Appl Phys Lett 2008;92(21):213113.

[8] Lepro X, Lima MD, Baughman RH. Spinnable carbon nanotubes forests grown on thin, flexible metallic substrates. Carbon 2010;48(12):3621-3627.

[9] Patole SP, Kim H-I, Jung J-H, Patole AS, Kim H-J, Han I-T, et al. The synthesis of vertically-aligned carbon nanotubes on an aluminum foil laminated on stainless steel. Carbon 2011;49(11):3522-3528.

[10] Wardle BL, Saito DS, Garcia EJ, Hart AJ, de Villoria RG, Verploegen EA. Fabrication and characterization of ultrahigh-volume fraction aligned carbon nanotube-polymer composites. Adv Mater 2008;20(14):2707-2714.

[11]Zhang M, Fang S, Zakhidov AA, Lee SB, Aliev AE, Williams CD, et al. Strong, transparent, multifunctional, carbon nanotube sheets. Science 2005;309(5738):1215-1219.

[12] Inoue Y, Suzuki Y, Minami Y, Muramatsu J, Shimamura Y, Suzuki K, et al. Anisotropic carbon nanotube papers fabricated from multiwalled carbon nanotube webs. Carbon 2011;49(7):2437-2443. 
[13] Pohls JH, Johnson MB, White MA, Malik R, Ruff B, Jayasinghe C, et al. Physical properties of carbon nanotube sheets drawn from nanotube arrays. Carbon 2012;50(11):4175-4183.

[14] Bradford PD, Wang X, Zhao H, Maria JP, Jia Q, Zhu YT. A novel approach to fabricate high volume fraction nanocomposites with long aligned carbon nanotubes. Compos Sci Technol 2010;70(13):1980-1955.

[15] Ogasawara T, Moon SY, Inoue Y, Shimamura Y. Mechanical properties of aligned multi-walled carbon nanotube/epoxy composites processed using a hot-melt prepreg method. Compos Sci Technol 2011;71(16):1826-1833.

[16] Wang Z, Yong ZZ, Li QW, Bradford PD, Liu W, Tucker DS, et al. Ultrastrong, stiff and multifunctional carbon nanotube composites. Mater Res Lett 2013;1:1925 .

[17] Jiang Q, Wang X, Zhu Y, Hui D, Qui Y. Mechanical, electrical and thermal properties of aligned carbon nanotube/polyimide composites. Compos Part B Eng $56: 408-12$

[18] Fisher FT, Bradshaw RD, Brinson LC. Effects of nanotube waviness on the modulus of nanotube-reinforced polymers. Appl Phys Lett 2002;80(24):4647-4649.

[19] Yazdchi K, Salehi M. The effects of CNT waviness on interfacial stress transfer characteristics of CNT/polymer composites. Compos Part A 2011;42(10):13011309.

[20] Dastgerdi JN, Marquis G, Salimi M. The effect of nanotubes waviness on mechanical properties of CNT/SMP composites. Compos Sci Technol 2013;86:164-169. 
[21] Tsai CH, Zhang C, Jack DA, Liang R, Wang B. The effect of inclusion waviness and waviness distribution on elastic properties of fiber-reinforced composites. Compos Part B Eng 2011;42(1):62-70.

[22] Joshi UA, Sharma SC, Harsha SP. Effect of carbon nanotube orientation on the mechanical properties of nanocomposites. Compos Part B Eng 2012;43: 2063-71.

[23] Cheng QF, Bao J, Park J, Liang Z, Zhang C, Wang B. High mechanical performance composite conductor: multi-walled carbon nanotube sheet/bismaleimide nanocomposites. Adv Funct Mater 2009;19(20):3219-3225.

[24] Wang X, Bradford PD, Liu W, Zhao H, Inoue Y, Maria JP, et al. Mechanical and electrical property improvement in CNT/Nylon composites through drawing and stretching. Compos Sci Technol 2011;71(14):1677-1683.

[25] Nam TH, Goto K, Oshima K, Premalal V, Shimamura Y, Inoue Y, et al. Effects of stretching on mechanical properties of aligned multi-walled carbon nanotube/epoxy composites. Composites Part A 2014;64:194-202.

[26] Nam TH, Goto K, Oshima K, Premalal EVA, Shimamura Y, Inoue Y, et al. Mechanical property enhancement of aligned multi-walled carbon nanotube sheets and composites through press-drawing process. Adv Compos Mater 2014, article in press and was online published http://dx.doi.org/10.1080/09243046.2014.985419.

[27] Jorkama M, von Hertzen R. The mechanism of nip-induced tension in winding. J. Pulp Paper Sci 2002;28(8):280-284.

[28] Liu W, Zhang X, Xu G, Bradford PD, Wang X, Zhao H, et al. Producing superior composites by winding carbon nanotubes onto a mandrel under a poly(vinyl alcohol) spray. Carbon 2011;49(14):4786-4791. 
[29] Ji J, Sui G, Yu Y, Liu Y, Lin Y, Du Z, et al. Significant improvement of mechanical properties observed in highly aligned carbon-nanotube-reinforced nanofibers. J Phys Chem C 2009;113(12):4779-4785.

[30] Fischer JE, Zhou W, Vavro J, Llaguno MC, Guthy C, Haggenmueller R, et al. Magnetically aligned single wall carbon nanotube films: Preferred orientation and anisotropic transport properties. J App Phys 2003;93(4):2157-2163.

[31] Tsuda T, Ogasawara T, Moon SY, Nakamoto K, Takeda N, Shimamura Y, Inoue Y. Three dimensional orientation angle distribution counting and calculation for the mechanical properties of aligned carbon nanotube/epoxy composites. Composites Part A 2014;65:1-9. 
Fig. 1 - (a) Vertically aligned CNT array and an inserted FE-SEM image showing horizontally aligned CNTs. (b) A STEM image and diameter distribution of CNTs. Fig. 2 - Schematic showing processing of aligned CNT sheet using drawing, stretching, winding and pressing techniques.

Fig. 3 - FE-SEM micrographs showing microstructural morphologies of (a) pristine, (b) stretched, (d) pressed, and (d) stretch-pressed aligned CNT sheets corresponding to CNT sheet processing methods of drawing and winding [12,15,25], stretch-drawing and winding [16], drawing and press-winding [26], and stretch-drawing and press-winding [this study].

Fig. 4 - Polarized Raman spectra of pristine, stretched, pressed and stretch-pressed CNT sheets at $0^{\circ}$ and $90^{\circ}\left(0^{\circ}\right.$ corresponds to a configuration where the polarization direction of the laser light is parallel to the CNT alignment direction, whereas $90^{\circ}$ corresponds to a configuration in which the laser light polarization direction is perpendicular to the CNT alignment direction).

Fig. 5 - FE-SEM micrographs showing in-plane CNT distribution of (a) pristine, (b) stretched, (c) pressed, and (d) stretch-pressed CNT/epoxy composites.

Fig. 6- FE-SEM micrographs showing fracture surfaces of (a) pristine, (b) stretched, (c) pressed, and (d) stretch-pressed CNT/epoxy composites.

Fig. 7 - Relations of $\sigma / V_{f}$ (tensile strength/volume fraction) and of $E / V_{f}$ (elastic modulus/volume fraction) versus the G-band intensity ratio.

Fig. 8 - Histograms showing the frequency distribution of global orientation angles with respect to the axial direction of CNTs in the non-pressed and stretch-pressed composites. Fig. 9 - Percentage increases in tensile strength, elastic modulus and CNT volume fraction of the composites. 
Table 1 - CNT fractions in the composites estimated from TGA ...

Table $1-$ CNT fractions in the composites estimated from TGA results

\begin{tabular}{lcccccc}
\hline \multicolumn{1}{c}{ Materials } & $\begin{array}{c}\text { Epoxy } \\
\text { resin }\end{array}$ & CNTs & $\begin{array}{c}\text { Pristine } \\
\text { composites }\end{array}$ & $\begin{array}{c}\text { Stretched } \\
\text { composites composites }\end{array}$ & $\begin{array}{c}\text { Pressed } \\
\text { composites }\end{array}$ \\
\hline Mass loss ${ }^{*}(\%)$ & 86.3 & 3.50 & 31.5 & 30.2 & 29.0 & 28.5 \\
$\begin{array}{l}\text { CNT mass } \\
\text { fraction (\%) }\end{array}$ & - & - & 66.2 & 67.8 & 69.3 & 69.9 \\
$\begin{array}{l}\text { CNT volume } \\
\text { fraction (vol. \%) }\end{array}$ & - & - & 60.1 & 61.3 & 62.9 & 63.4 \\
\hline
\end{tabular}

${ }^{*}$ Mass loss was measured between $150^{\circ} \mathrm{C}$ and $750^{\circ} \mathrm{C}$. 
Table 2 - Properties of pristine, stretched, pressed and stretch-pressed CNT/epoxy composites

\begin{tabular}{|c|c|c|c|c|c|c|}
\hline Composite & $\begin{array}{l}\text { CNT sheet } \\
\text { processing }\end{array}$ & $\begin{array}{l}\text { Thickness } \\
(\mu \mathrm{m})\end{array}$ & $\begin{array}{l}\text { Density } \\
\left(\mathrm{g} / \mathrm{cm}^{3}\right)\end{array}$ & $\begin{array}{c}\text { Tensile strength } \\
(\mathrm{MPa})\end{array}$ & $\begin{array}{c}\text { Elastic } \\
\text { modulus (GPa) }\end{array}$ & $\begin{array}{c}\text { Fracture strain } \\
(\%)\end{array}$ \\
\hline Pristine & $\begin{array}{l}\text { Drawing and } \\
\text { winding }\end{array}$ & $21-24$ & 1.42 & $592.6 \pm 51.7$ & $105.5 \pm 10.6$ & $0.56 \pm 0.04$ \\
\hline Stretched & $\begin{array}{l}\text { Stretch-drawing } \\
\text { and winding }\end{array}$ & $17-20$ & 1.44 & $674.5 \pm 63.6$ & $117.9 \pm 11.5$ & $0.57 \pm 0.04$ \\
\hline Pressed & $\begin{array}{l}\text { Drawing and } \\
\text { press-winding }\end{array}$ & $16-18$ & 1.45 & $724.4 \pm 60.3$ & $126.1 \pm 11.4$ & $0.58 \pm 0.06$ \\
\hline $\begin{array}{l}\text { Stretch- } \\
\text { pressed }\end{array}$ & $\begin{array}{l}\text { Stretch-drawing } \\
\text { an press-winding }\end{array}$ & $16-18$ & 1.46 & $780.2 \pm 71.2$ & $133.6 \pm 13.4$ & $0.59 \pm 0.06$ \\
\hline
\end{tabular}


Table 3 - Intensity ratio $\left(I_{\mathrm{G}} / I_{\mathrm{D}}\right)$ and G-band intensity ratio $(R)$ of the composites

\begin{tabular}{|c|c|c|c|c|}
\hline \multirow{2}{*}{$\begin{array}{l}\text { Composite } \\
\text { sample }\end{array}$} & \multirow{2}{*}{$\begin{array}{l}\text { CNT sheet } \\
\text { processing }\end{array}$} & \multicolumn{2}{|c|}{ Intensity ratio $\left(I_{\mathrm{G}} / I_{\mathrm{D}}\right)$} & \multirow{2}{*}{$\begin{array}{c}\text { G-band intensity } \\
\text { ratio }(R)\end{array}$} \\
\hline & & $0^{\circ}$ & $90^{\circ}$ & \\
\hline Pristine & $\begin{array}{l}\text { Drawing and } \\
\text { winding }\end{array}$ & 2.82 & 3.48 & 1.38 \\
\hline Stretched & $\begin{array}{l}\text { Stretch-drawing } \\
\text { and winding }\end{array}$ & 2.89 & 3.46 & 1.73 \\
\hline Pressed & $\begin{array}{l}\text { Drawing and } \\
\text { press-winding }\end{array}$ & 2.92 & 3.50 & 1.98 \\
\hline $\begin{array}{l}\text { Stretch- } \\
\text { pressed }\end{array}$ & $\begin{array}{l}\text { Stretch-drawing } \\
\text { an press-winding }\end{array}$ & 2.94 & 3.54 & 2.24 \\
\hline
\end{tabular}


Fig. 1 - (a) Vertically aligned CNT array and ...

a

$10 \mathrm{~mm}$
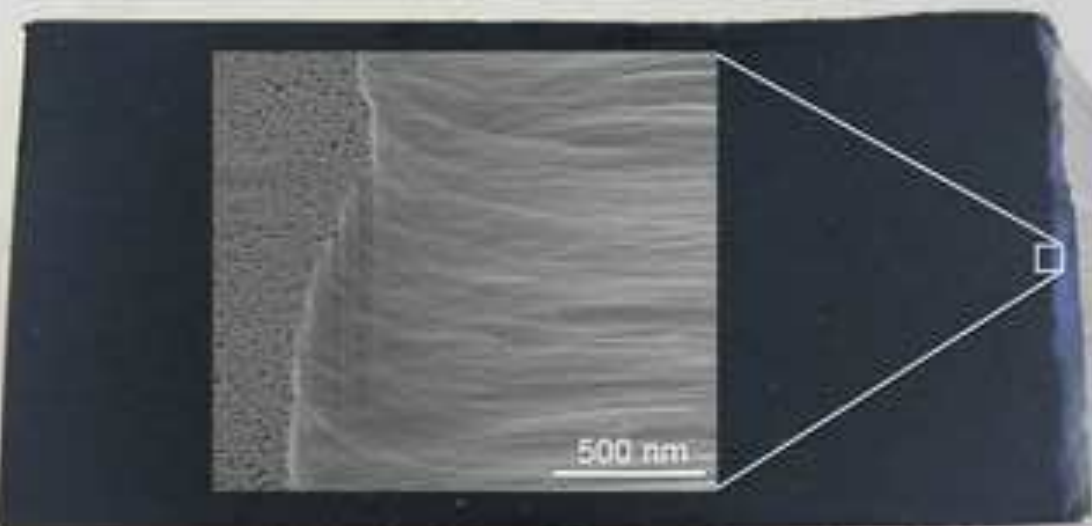
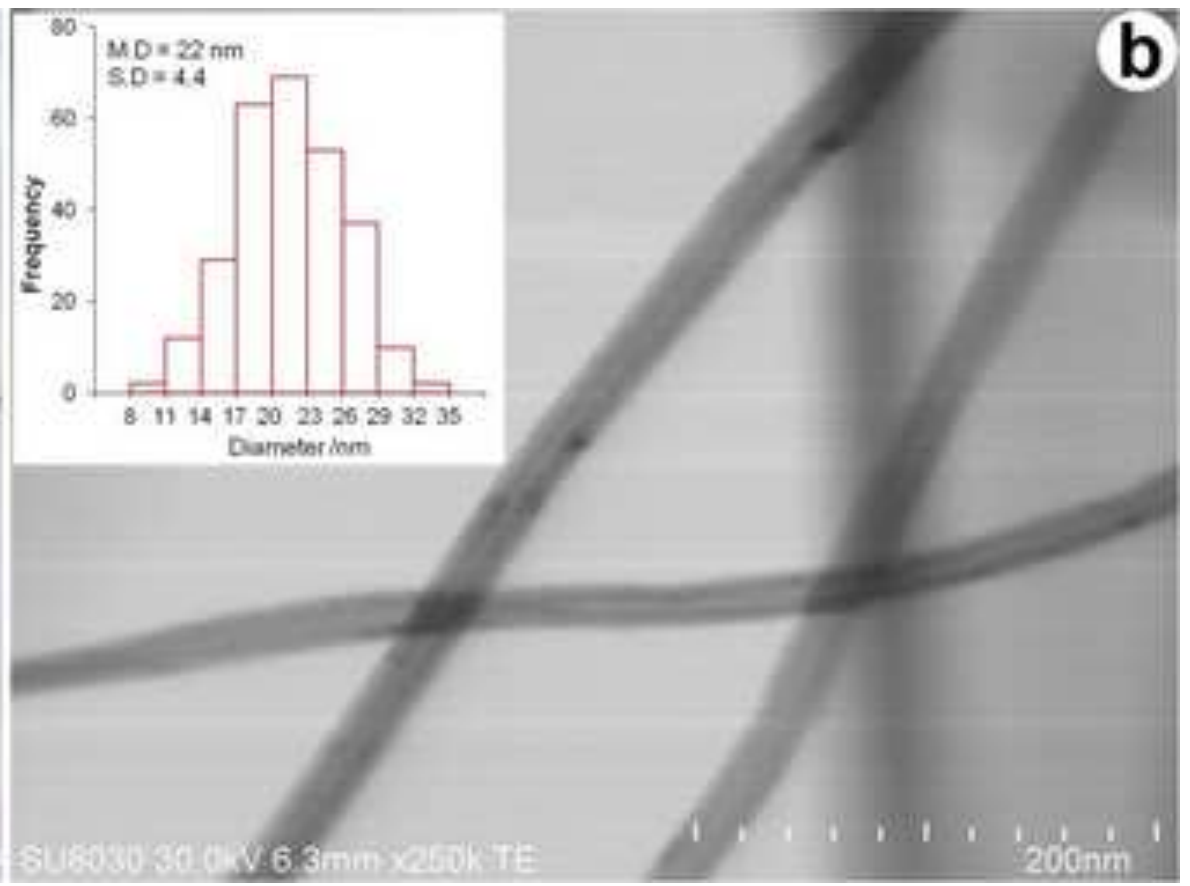


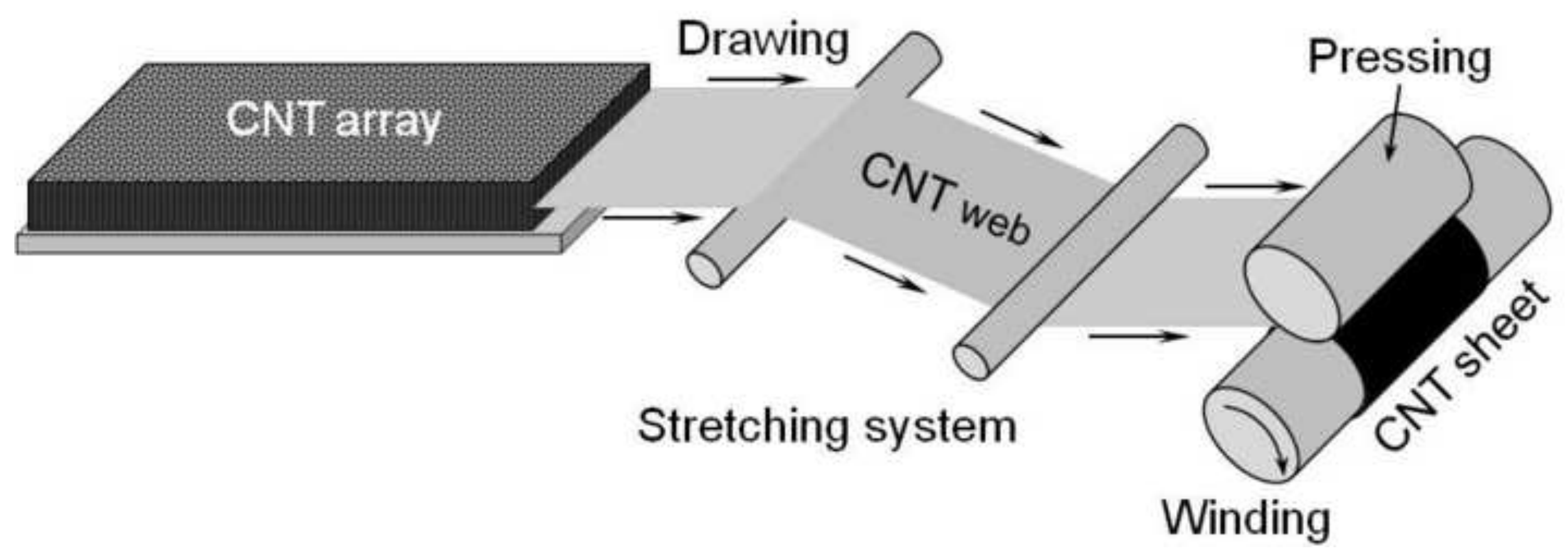


Fig. 3 - FE-SEM micrographs showing microstructural ...
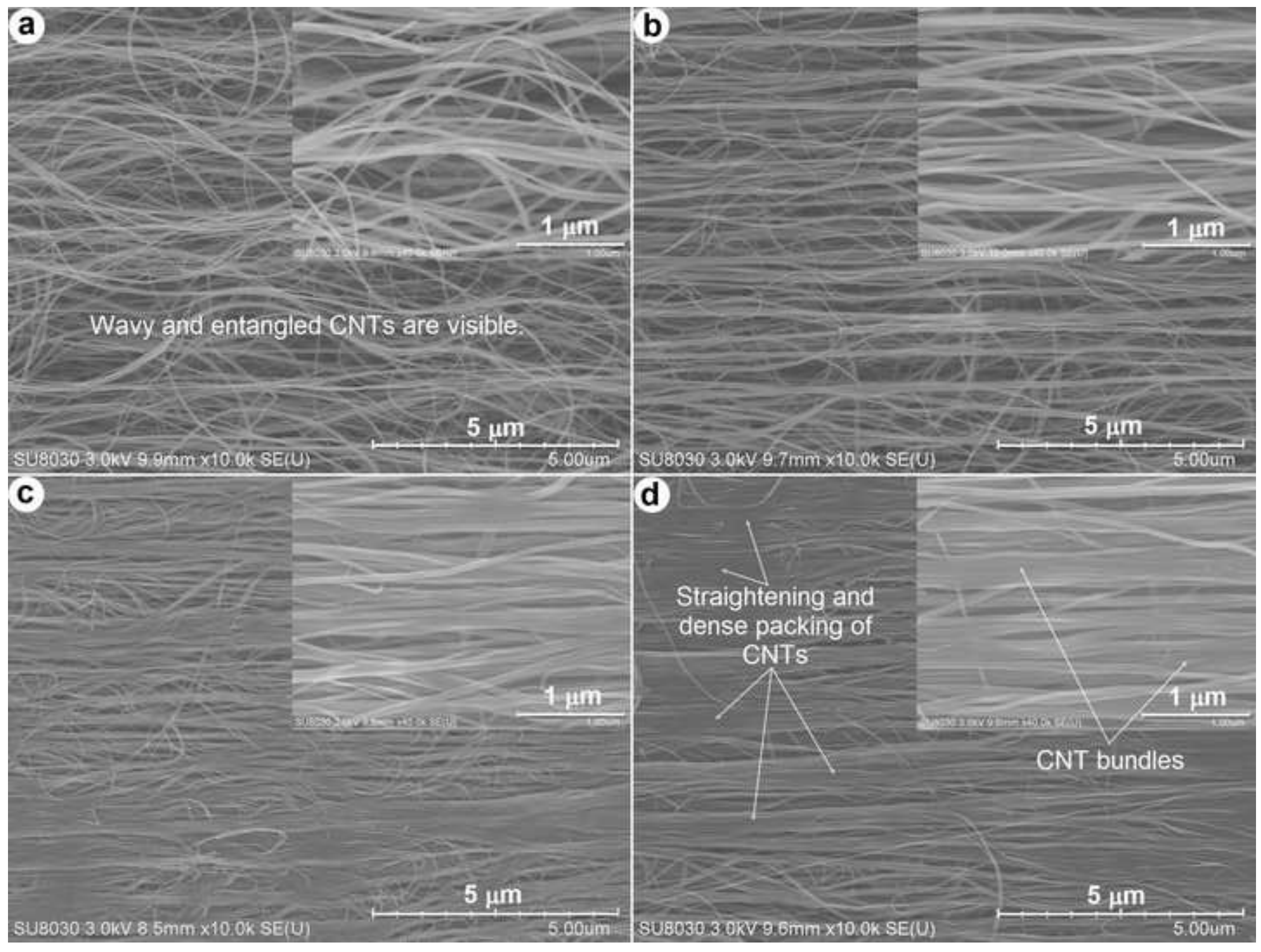
Fig. 4 - Polarized Raman spectra of pristine, stretched, ...

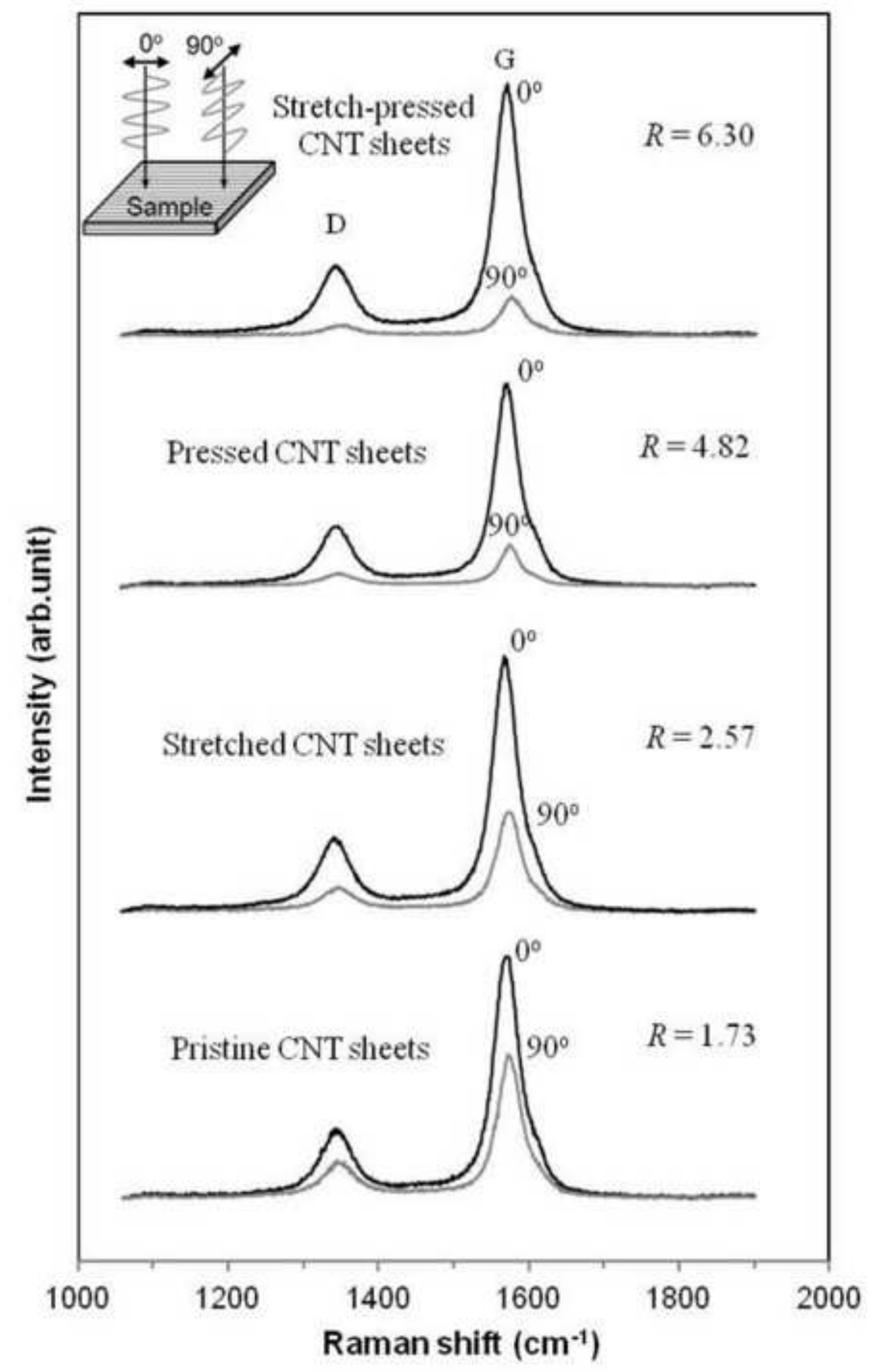


Fig. 5 - FE-SEM micrographs showing in-plane CNT ...

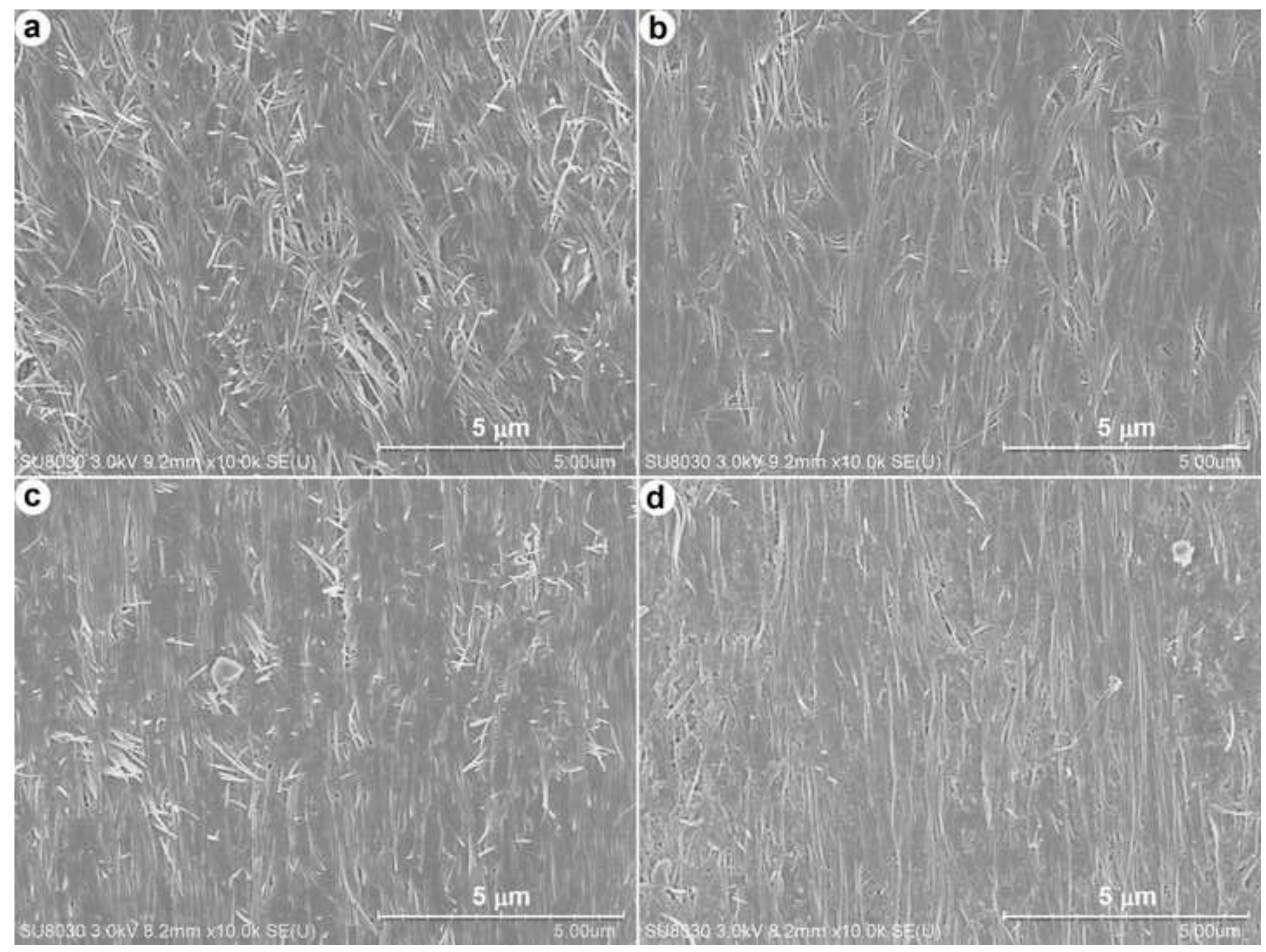




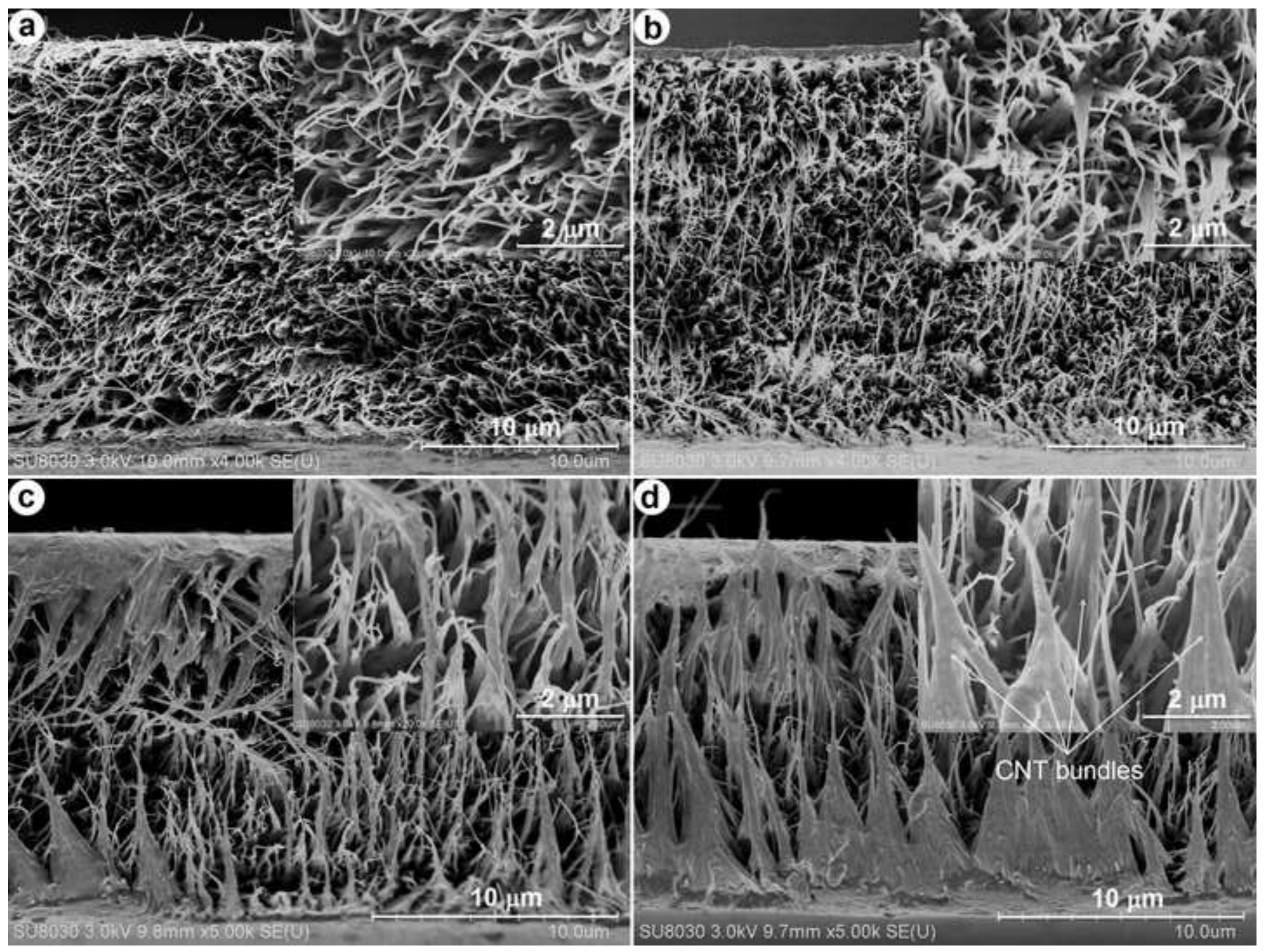




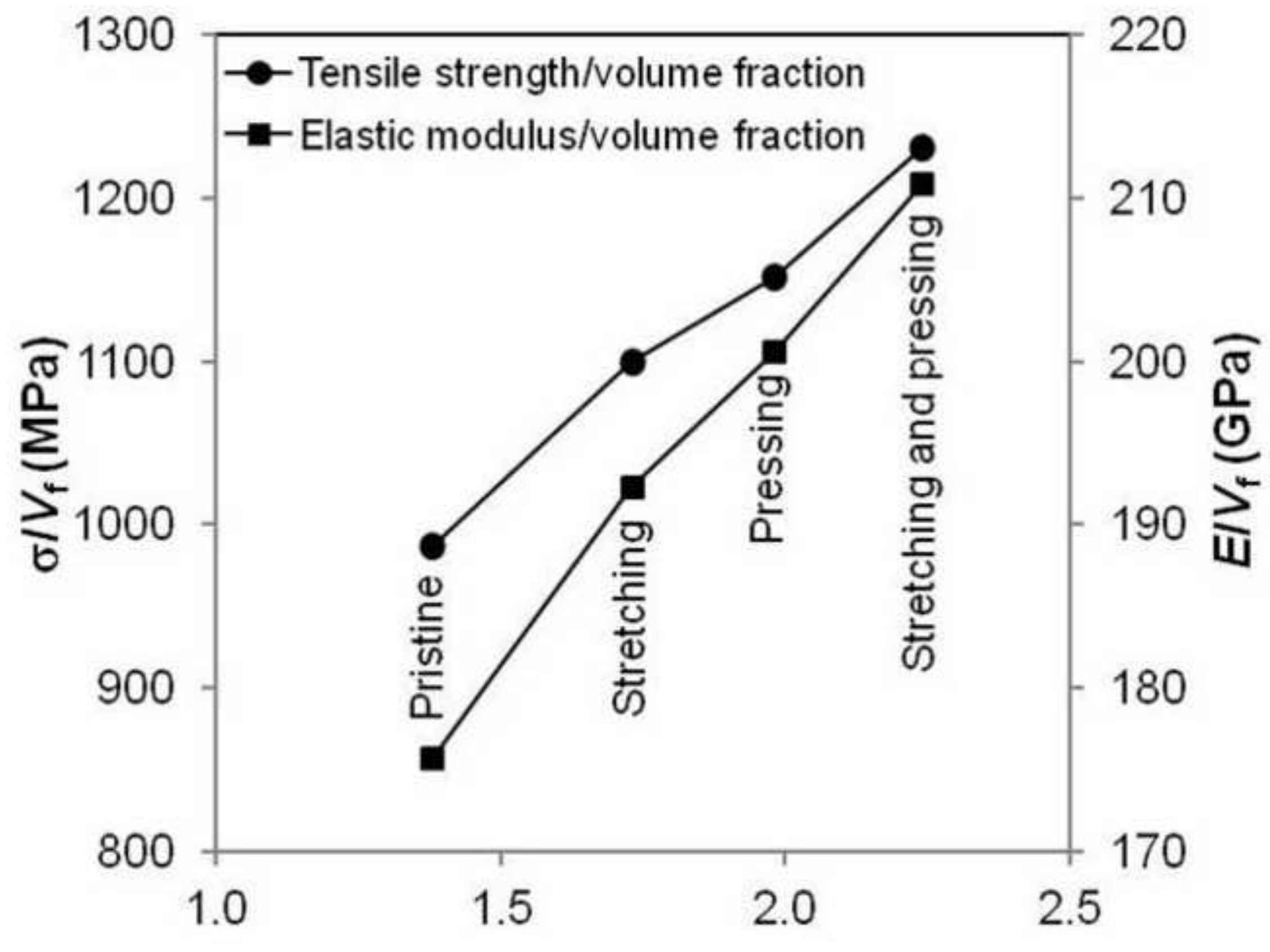

G-band intensity ratio $R$ 


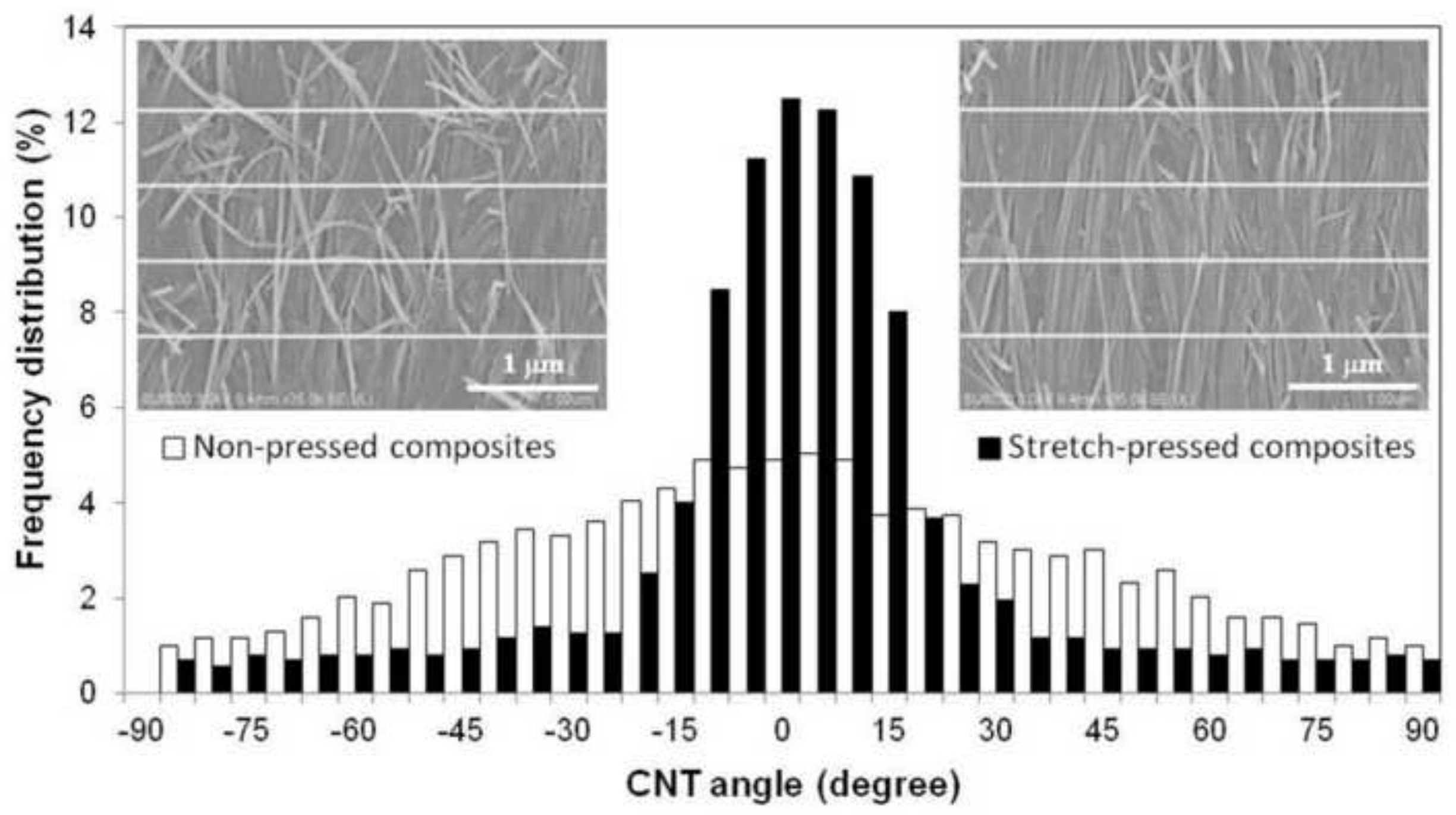




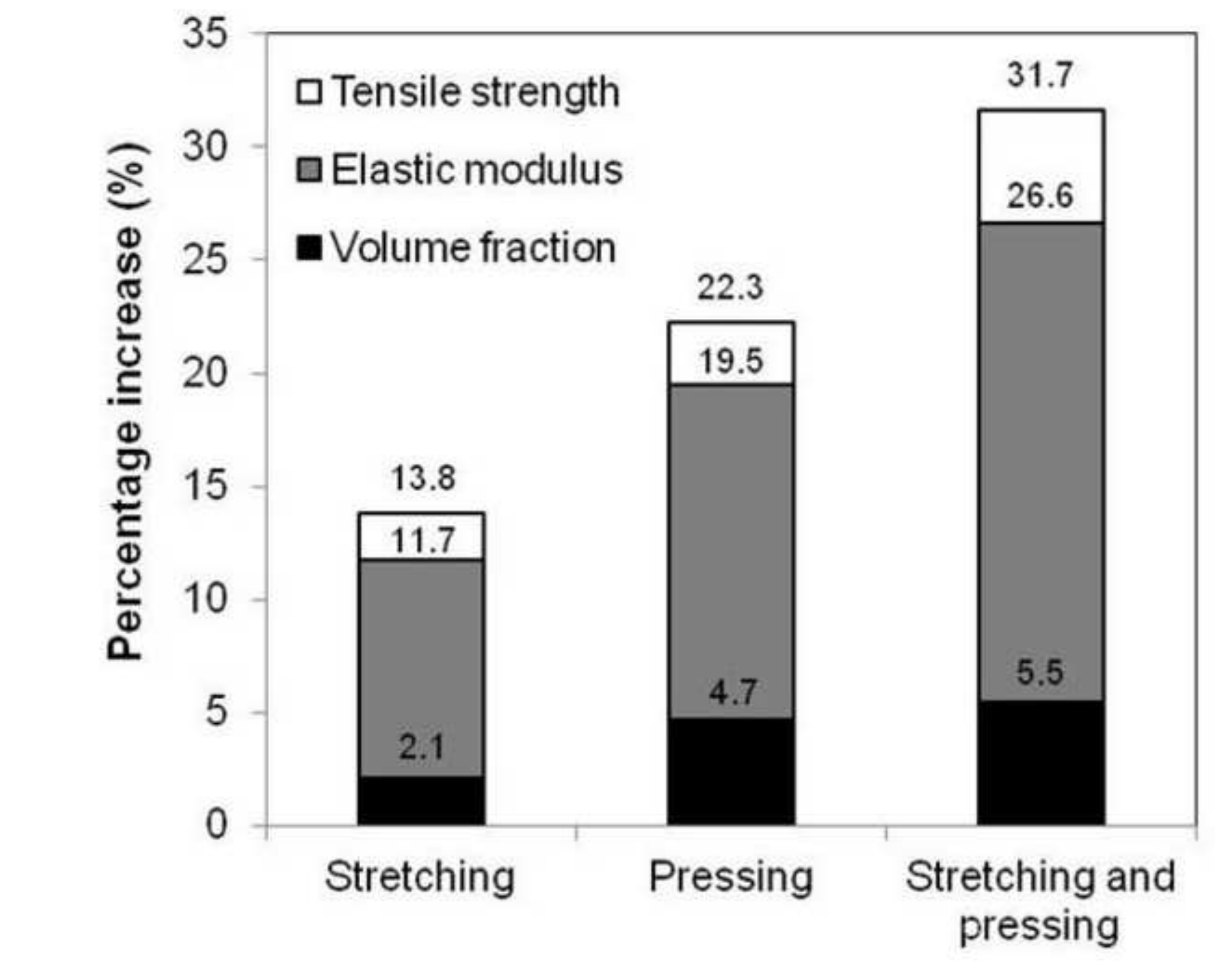

Fig. 9 - Percentage increases in tensile strength, ...
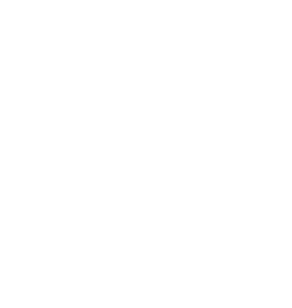

.

